\title{
The Distribution, Relationships to Other Organelles, and Calcium-sequestering Ability of Smooth Endoplasmic Reticulum in Frog Olfactory Axons
}

\author{
PAUL R. BURTON ${ }^{2}$ AND LEIGH A. LAVERI \\ Department of Physiology and Cell Biology and the Center for Biomedical Research, University of Kansas, Lawrence, Kansas 66044
}

\begin{abstract}
Ultrastructural studies of single and serial sections of bullfrog olfactory axons showed that smooth endoplasmic reticulum (SER) tubules, which usually appear as single profiles in cross-sections of axons, are continuous over considerable distances, but that discontinuities do exist. Computer-assisted three-dimensional reconstruction of portions of axons indicated that the SER tubules show considerable variation in the volume of the cisternal space along the tubule, which often follows a tortuous path. Some branching and anastomosing appears to occur, and electron-dense material was present in the cisternal space of some tubules. SER tubules are often bridged to neurofilaments and less often to microtubules. The usual two to three microtubules in the axoplasm form a domain which is characterized by a clear area, or zone of exclusion, around the microtubules. Ultrastructural cytochemistry was used to demonstrate that SER tubules actively sequester $\mathrm{Ca}$. The electron-dense product (calcium oxalate) was uniformly and specifically associated with the SER of axons at both proximal (closest to the perikarya in the olfactory epithelium) and distal (closest to the olfactory lobe of the brain) ends of the olfactory nerve. It is concluded that the primary function of SER tubules in these axons is to serve in the regulation of $\mathrm{Ca}$ in the axoplasm, probably to facilitate fast axoplasmic transport, and that a secondary function may be the translocation of material in the cisternal space. The observations are discussed as they may relate to the "microstream" hypothesis of axoplasmic transport, and it is argued that fast transport occurs through the zone of exclusion associated with the microtubule domain(s) of axons.
\end{abstract}

The predominant formed elements of typical vertebrate axons include microtubules, neurofilaments, mitochondria, smooth endoplasmic reticulum (SER), and, in many instances, various vesicular and vacuolar elements. These components are often seen to be interconnected with one another by a matrix of delicate filaments. Axoplasmic transport of components, from molecules to mitochondria, occurs at several rates and in both directions through this mileu. In addition to the axoplasmic matrix space defined by the

Received February 11, 1985; Revised April 15, 1985;

Accepted April 16, 1985

\footnotetext{
${ }^{1}$ This work was supported by Public Health Service Grant GM 24992 and a grant from the University Biomedical Science Support Grant Subcommittee. We wish to thank our colleagues, Drs. William Dentler and Daryl Hartter for critically reading the manuscript and offering helpful suggestions.

${ }^{2}$ To whom correspondence should be sent.
}

limiting axolemma, two other closed membrane compartments are always present. One compartment is that which occurs within mitochondria, which is actually a double compartment, and the other is the cisternal space of the SER.

Ducros (1974) examined semi-serial sections of SER in large axons of Octopus nerve and attempted to reconstruct its threedimensional (3-D) nature. The SER was described as a peripheral tubular network continuous with a more central system of "irregularly anastomosing tubules with varicosities." It was suggested that the SER might play a role in translocation of metabolites. Earlier, Lieberman (1971) had described an intimate spatial relationship between SER and microtubules in frog neurons and had theorized that neuronal SER might function as does sarcoplasmic reticulum in sequestering $\mathrm{Ca}$. The high voltage electron microscope was used to examine axons in thick sections of nerves secondarily fixed in the presence of potassium ferrocyanide (after diaminobenzidine incubation) to better visualize membrane elements, including axonal SER (Tsukita and Ishikawa, 1976); in this study, the SER was determined to be a continuous network of narrow, longitudinally oriented tubules that anastomosed with each other.

Droz et al. (1975) studied the spatial arrangement of SER in axons of rat spinal and chick ciliary ganglia and concluded that the SER formed a continuous system extending the length of the axon. Furthermore, ultrastructural radioautography provided evidence that labeled proteins might be rapidly translocated in association with SER, even though the location of silver grains made it impossible to determine whether labeled proteins were located within the cisternae or on the surface of the reticulum. Other workers examined the ultrastructure of axons on either side of locally chilled sites alongnerves, with chilling sufficient to block axoplasmic transport. Membranous elements accumulated in axons on the proximal side of the chilled sites, and these "vesicotubular" membranes were considered to bo SER, or derived from SER (Tsukita and Ishikawa, 1979, 1980).

Using a cytochemical method to localize $\mathrm{Ca}$, with calcium oxalate as an electron-dense product, along with x-ray microanalysis, Henkart et al. (1978) provided the first convincing evidence that the SER of axons could sequester $\mathrm{Ca}$. Since then, the pumping and sequestering of Ca by SER has been confirmed in many kinds of axons (e.g., Duce and Keen, 1978; McGraw et al., 1980; Chan et al., 1984), in perikarya and dendritic spines (Henkart, 1980; Fifkova et al., 1983), and in invertebrate and vertebrate photoreceptors (Walz, $1979,1982 \mathrm{a}, \mathrm{b}, \mathrm{c}$; Ungar et al., 1984). The accumulation of Ca by axonal SER is an energy-dependent process, one of several properties shared by SER and the sarcoplasmic reticulum of muscle cells (Walz, 1983).

In terms of translocation of material in association with axonal SER, three obvious possibilities come to mind: (1) material could become linked to the SER membrane and be transported along with membrane flow (conveyer belt hypothesis; see Hammerschlag and 
Stone, 1982); (2) material could gain access to the cisternum, perhaps even in the perikaryon, as a consequence of translation of proteins, and then be translocated through the cisternal space (conduit hypothesis; see Teichberg and Holtzman, 1973; Alonso and Assenmacher, 1979); and, (3) material sequestered in the cisternum could be trapped as cargo in vesicles pinching off the SER, be transported through the axoplasm for a given distance (perhaps along the surface of microtubules), and then fuse with a main SER tubule, returning the material to the cisternal space (shuttle hypothesis; see Tsukita and Ishikawa, 1976).

The literature reflects some uncertainty about whether axonal SER extends from the perikaryon to the axon terminal, and it is obvious that difficulties have arisen in distinguishing SER membranes from other membranous elements in the axoplasm, particularly in large axons. Also, possible associations between SER and other axoplasmic constituents have not been studied in any detail, and if the SER does serve as a conduit, why is there so little evidence of material in its cisternae? For example, if shuttle vesicles are continuously pinching off the main SER tubules for rapid translocation, then emptying their contents back into the cisternal space by membrane fusion, should there not be structural evidence for such activity? Furthermore, since one function of axonal SER is to sequester $\mathrm{Ca}$, it would be desirable to determine whether there are regional differences in this property along the axon.

Frog olfactory axons are small and structurally uncomplicated; in many respects they are ideal model systems for ultrastructural studies of axons. Cross-sections of these axons show that they contain one, or a few, SER profiles, and that, except for mitochondria, no other membranous elements are normally present in the axoplasm. In addition to an SER tubule and an occasional mitochondrion, the only other prominent formed elements are a few microtubules (usually two or three) and a variable but small number of neurofilaments. The structural simplicity of the olfactory axon led us to study its SER in considerable detail, and these studies provide evidence that: (1) the SER is continuous over great distances in the axon, but portions of some axons show no SER profiles at all; (2) the tubule of SER usually follows a tortuous path, often with great variations in its cisternat volume; (3) there is no structural evidence of lateral vesiculation or vesicle fusion and, in fact, vesiclos are not usually encountered in these axons; (4) dense material is sometimes seen in the cisternal space; (5) the SER is often bridged to neurofilaments or microtubules, but it seems to be more often associated with the former; and (6) SER of frog olfactory axons sequesters $\mathrm{Ca}$ by an ATP-dependent process, and the sequestration of $\mathrm{Ca}$ appears uniform along the SER at points proximal and distal to the perikaryon.

\section{Materials and Methods}

Adult bullfrogs (Rana catesbeiana) were obtained locally or from commercial sources, kept in an amphibian facility at $20^{\circ} \mathrm{C}$, and fed regularly. Isolation of olfactory nerve pieces into proximal and distal pieces, with reference to the perikarya in the olfactory epithelium, has been described and illustrated elsewhere (Burton, 1984). A number of fixatives were used, including $2.5 \%$ glutaraldehyde buffered with $0.1 \mathrm{M}$ cacodylate at $\mathrm{pH} 7.2$ to 7.4. Most of the material used in this study, however, was fixed in $2 \%$ glutaraldehyde in $0.1 \mathrm{M}$ PIPES buffer $(\mathrm{pH} 6.9)$ containing $10 \mathrm{~mm}$ EGTA, 2 mM $\mathrm{MgCl}_{2}$, and $0.5 \mathrm{~mm}$ guanosine $5^{\prime}$-triphosphate. Fixation was usually for $1 \mathrm{hr}$ at room temperature with continuous agitation. Thereafter material was secondarily fixed in $1 \%$ osmium tetroxide buffered with $0.1 \mathrm{M}$ cacodylate ( $\mathrm{pH} 7.2$ to 7.4 ), processed with or without staining in $2 \%$ aqueous uranyl acetate for 30 to $60 \mathrm{~min}$, dehydrated in acetone, and embedded in Araldite 502 resin.

Silver to gray sections were obtained with a diamond knife, picked up on 400 mesh grids, and typically stained with methanolic-ethanolic uranyl acetate and lead citrate (10 min and $5 \mathrm{~min}$, respectively; see Kim et al., 1979). Sections were examined in a Philips 300 transmission electron microscope. Serial sections were obtained using a simple micromanipulator as a "third hand," following the methods described in detail by Rieder (1981). Ribbons of 10 to 20 wrinkle-free sections of uniform appearance were picked up on Formvar-and carbon-coated grids wilt $0.4 \times 2$ rum slots, then stained with aqueous uranyl acetate and lead citrate in a conventional manner.
Serial cross-sections of axons were used to obtain computer-assisted 3 D reconstructions of axoplasmic constituents, including SER; the serial sections were estimated to be $70 \mathrm{~nm}$ thick. Serial micrographs of groups of axons were obtained at magnifications of $\times 54,000$, and subgroups of axons were selected and enlarged five times to provide a final print magnification of $\times 270,000$. Cross-sectional profiles of axoplasmic components were then sequentially digitized and the tracings stored in the memory of an IBM personal computer with 256K RAM interfaced with a TallGrass $12.5 \mathrm{Mb}$ Winchester hard disk. Using an image reconstruction program written for the University of Kansas, profiles of axoplasmic elements could be displayed on a monitor in different colors in a stacked configuration (i.e., in register). Furthermore, the stacked images could be rotated in space to provide various perspective views, with any or all of the structures digitized displayed on the screen. When a particular perspective view was found to be informative, the compositc image was plotted, or reconstructed, in color using an Hewlett Packard 7470A plotter. For stereoscopic viewing in true 3-D, two views of a given composite reconstruction were plotted rotated (tilted) by $5^{\circ}$ with respect to each other. The slereoscopic pairs are mounted such that they can be viewed using an Abrams CF 8 stereoscopic viewer or by individuals experienced in crossing the eyes to provide fusion of the two sets of images. Hurther details on the 3-D reconstructions will be provided in the text as appropriate, and the methods and computer program used are described in detail elsewhere (Burton, 1985).

The cytochemical methods of Walz $(1979,1982 a)$ were used to localize $\mathrm{Ca}$ sequestered by SER, with the methods modified from those originally described by Heumann and Zebe (1967). Fssentially, the approach involved brief incubation of fresh nerve pieces in a lysis medium containing saponin $(500 \mu \mathrm{g} / \mathrm{ml})$, which selectively permeabilizes cholesterol-rich plasma membranes but not endomembranes such as SER within the incubation time used. Material was then incubated in a loading medium containing ATP and potassium oxalate (at a calculated $0.85 \mu \mathrm{M} \mathrm{Ca}$ ), and membrane compartments competent to actively sequester $\mathrm{Ca}$ at this concentralion accumulated the ion. Under these conditions, the level of $\mathrm{Ca}$ in the compartment rises to the critical concentration required to complex with oxalate, forming electrondense calcium oxalate which remains at or near the site of its formation. Thereafter, nerve pieces were fixed and prepared for electron microscopy.

Pieces of the proximal (to the perikarya) and distal (closest to the olfactory lobe of the brain) olfactory nerve were incubated in lysis medium for $15 \mathrm{~min}$ at room temperature and then transferred to loading medium and incubated at room temperature (or at 0 to $4^{\circ} \mathrm{C}$ with no apparent difference) for $60 \mathrm{~min}$. Thereafter, samples were fixed in $2.5 \%$ glutaraldehyde in $0.1 \mathrm{M}$ cacodylate butfer ( $\mathrm{pH} 7.2$ to 7.3 ) containing $40 \mathrm{~mm}$ potassium oxalate; fixation was at 0 to $4^{\circ} \mathrm{C}$ for $60 \mathrm{~min}$. Material was rinsed in buffer containing $40 \mathrm{~mm}$ potassium oxalate and fixed for 60 min at room temperature in $1 \%$ osmium tetroxide buffered with $0.1 \mathrm{M}$ cacodylate, also containing potassium oxalate. Nerve pieces were then dehydrated in an acetone series and embedded in Araldite 502 resin. Sections were examined unstained and were obtained using $10 \%$ ethanol in the knife trough to which was added $40 \mathrm{~mm}$ potassium oxalate. Upon picking up ribbons on 400 mesh uncoated grids, the grids were allowed to dry and then were gently rinsed with distilled water to remove any excess oxalate from the surface of the sections. For control material, nerve pieces were permeabilized and incubated without ATP, in the presence of 1 $\mathrm{mg} / \mathrm{ml}$ of apyrase to inhibit ATP activity, or in the absence of potassium oxalate.

\section{Results}

Typical olfactory axons of the frog are shown in cross-section in Figure 1. Their diameter usually falls in the $0.1-$ to $0.3-\mu \mathrm{m}$ range and their most prominent feature is their small size; the most prominent organelles in the axoplasm are microtubules (usually two or three in number), one or more profiles of SER (usually one is seen), a variable number of neurofilaments, and an occasional mitochondrial profile. Often the neurofilaments occur in a discrete group, as in the two axons in Figure 1, and lateral bridges between their roughly circular profiles are often evident, as between two pairs of neurofilaments in the lower axon. The microtubules also often appear to occupy a defined area of the axoplasm. The third obvious profile in most cross-sections is that of SER, which is highly variable in its shape and size, the latter quality reflecting the luminal (cisternal) volume of the compartment. The irregularly shaped SER profiles appear to be more often associated with neurofilaments than with microtubules.

In favorable axonal cross-sections, the filamentous nature of the axoplasmic matrix is evident, as shown in the lower axon in Figure 


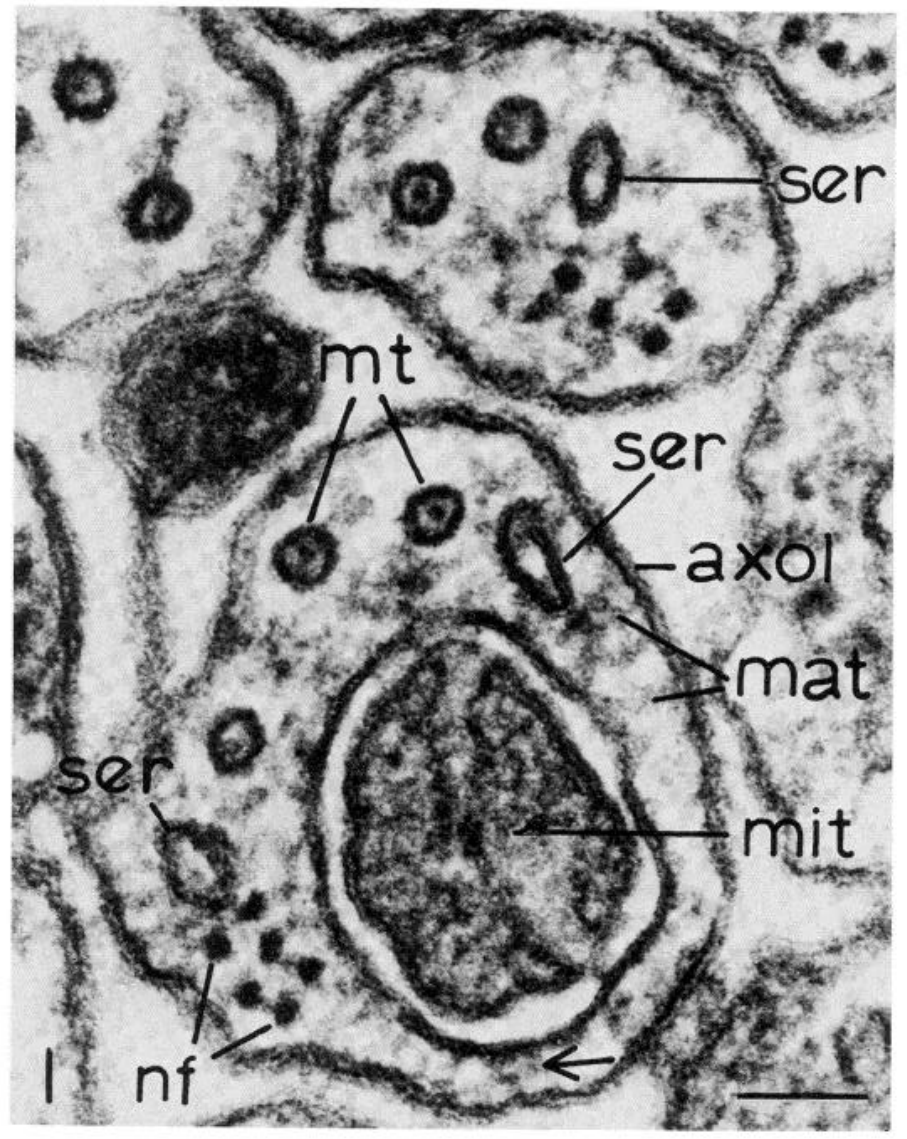

Figure 1. Frog olfactory axons seen in cross-section. In the lower axon, two profiles of smooth endoplasmic reticulum (ser) can be seen. A mitochondrial (mit) profile is apparent, along with three microtubules $(m t)$ and six neurofilaments $(n f)$. Wispy axoplasmic matrix (mat) filaments are mainly associated with the SER, mitochondrial, and neurofilament profiles; filaments extend from the lower surface of the mitochondrion (arrow) to connect it to the inner surface of the axolemma $(a x o l)$. Note the close association between the SER profile at the lower left and the six neurofilaments; two pairs of the neurofilaments are bridged together. In the axon at the top, the neurofilaments occur as a group enmeshed in a vague matrix network, with the SER profile nearby. Magnification $\times 264,000 ;$ bar $=0.05 \mu \mathrm{m}$.

1. In this axon, the matrix is most evident in its association with SER, neurofilaments, and the mitochondrion, where filaments can be seen (Fig. 1, arrow) connecting the outer mitochondrial membrane to the inner surface of the axolemma (Fig. 1, axol). Often an irregular meshwork of matrix filaments is associated with an SER-neurofilament complex. In contrast, as shown in Figure 1, a filamentous matrix is often absent around microtubule profiles, and it appears that the microtubules and the empty-appearing area around them comprise another axoplasmic domain.

Studies of longitudinal profiles show that the SER extends uninterrupted for considerable distances within olfactory axons. In the axon at the top of Figure 2, a tubule of SER can be seen extending across the micrograph; at the left (Fig. 2, arrow), the SER tubule is linked to one of the two microtubules in the axon, and along its lower surface it is bridged to the axolemma by matrix material. In this same axon, the matrix-free channels along the microtubules can be clearly seen, even though at several points the upper microtubule is bridged to the axolemma (Fig. 2, top arrow), and a bridge or two can also be seen between the microtubules themselves. Studies of microtubules in longitudinal views of axons indicate that they are relatively straight structures. In the axon at the bottom of Figure 2 , the SER shows an apparent blind end at the left and a region where the cisternal space (Fig. 2, cist) is greatly expanded and follows the contour of the axolemma. At the left end the SER is associated with a neurofilament, which has the characteristic "pipe cleaner" appearance with many lateral filaments extending from its surface. At the lower left in Figure 2 the neurofilament is linked (Fig. 2, arrow) to another tubule of SER seen in tangential section.

Figures 3 to 5 all show portions of single axons in longitudinal section. Wispy filaments can be seen extending from the surface of the SER in Figure 3 (arrows), and at the top, some of these filaments link the SER to the axolemma. At the top of Figure 4, two neurofilaments are seen to be interconnected by bridges, and the lower neurofilament is also clearly linked to the SER. The blind end of an SER tubule is shown at the right in Figure 5 (arrow), and the great variation in the dimensions of the cisternal space (Fig. $5, x$ ) is well illustrated. A neurofilament is closely associated with the upper surface of the SER profile.

It is not uncommon to see material in the cisternae of SER, as shown in Figures 6 to 11. Sometimes the material appears as a small, compact mass centrally located in the cisternum (Figs. 6 and 7). In other cases the cisternal material is more amorphous (Figs. 8 to 11). Filamentous elements are seen to be associated with the surface of SER profiles in Figures 6 and 7 (arrows), and Figure 11 (open arrowheads to the right); two profiles of SER are seen in Figure 11 (arrows), only one of which shows cisternal material. Infrequently, SER will contain large, dense masses of material, as shown in Figure 15, where serial sections of an axon were obtained. In this axon, the SER was observed to branch and anastomose, as will be discussed later, but one of the profiles in the series contains a mass of electron-dense material which appears to be roughly spherical. Even though some material is seen in SER profiles in Figure $15, f$ and $g$, the primary mass is seen in Figure $15, j$ to $m$, where it appears to have a large dense core limited by what may be one or more trilaminar membranes (Fig. 15, $k$ and $l$ ).

To obtain additional information on the 3-D path of SER through the axoplasm, up to 20 serial sections were obtained of many axons. Figure 12 shows such a series, in which the SER (open arrowhead) is seen to follow a fairly straight path through the series and remains in a given axoplasmic area, although the shape and dimensions of the SER profiles vary somewhat from section to section. In this series, there is an obvious association between the SER and neurofilaments. To further visualize the SER in this axon in three dimensions, axoplasmic components were digitized and then subjected to computer-assisted reconstruction (Fig. 13). Figure 13a shows the complete reconstruction of the axon for 15 sections (representing approximately $1 \mu \mathrm{m}$ of axon length), along with the profiles of SER from an adjacent axon which were also digitized. The profiles are turned on edge vertically, or rotated $65^{\circ}$ (i.e., each slice or profile is turned $65^{\circ}$ around its $Y$-axis), and displayed in a series with the distance separating the profiles (sections) selected as appropriate. Note that the SER of the adjacent (lower) axon follows a more wandering path than the one at the top. In the complete reconstruction at the top, however, it is difficult to follow the profiles of SER because of overlapping profiles of other structures. To better delineate the 3-D paths of the SER in the two axons, only the SER and axolemma were selected for display, and in this instance stereoscopic pairs were plotted (again the profiles were rotated $65^{\circ}$ around the $Y$-axes, but the two plots differed by a $5^{\circ}$ rotation in the $X$-axis). This allows the viewer to visualize the separated profiles in true 3-D space (Fig. 13, $b$ and $c$ ). Clearly, the SER in the upper axon follows a relatively straight path whereas that of the lower axon is more wandering.

Serial sections of three olfactory axons are shown in Figure 14. A number of observations can be made based on this series. In the axon at the top, the SER profiles (open arrowheads) follow a relatively straight path through the axoplasm, even though the shapes of the profiles are somewhat variable from section to section. Again it is obvious that the SER is more closely associated with neurofilaments than with microtubules, and that an electron-lucent area can be delineated around the microtubule profiles. By following the three 

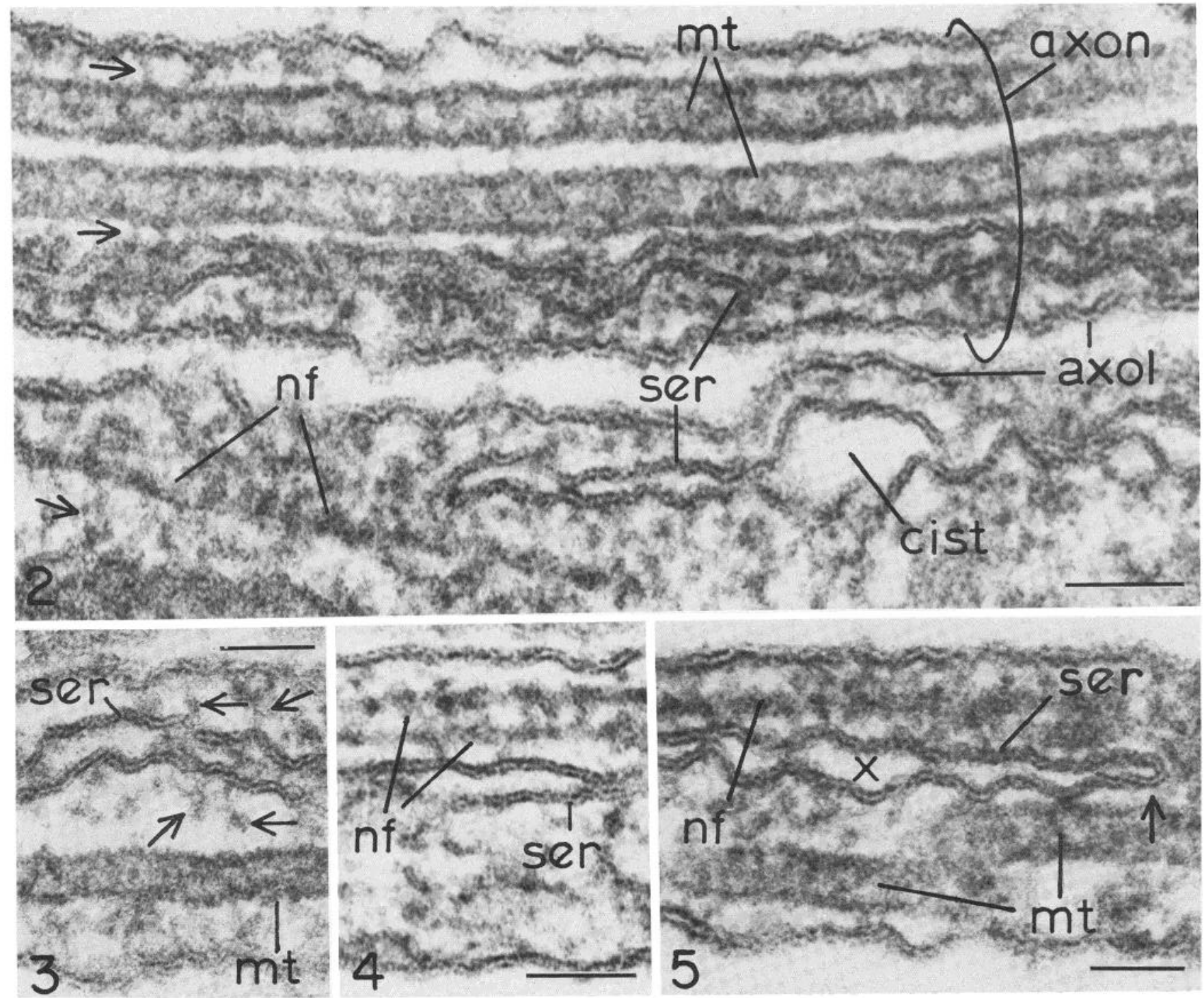

Figures 2 to 5. Longitudinal views of olfactory axons. A portion of an axon is shown at the top in Figure 2 in which an SER tubule and two microtubules $(m t)$ can be seen. A clear zone, or zone of exclusion, is clearly seen around the microtubules. Occasional bridges are seen between the microtubules themselves or between the microtubules and the axolemma (top arrow) or the SER tubule (lower arrow). The SER tubule extends across the micrograph and its irregular or wavy profile can be clearly seen. The lower aspect of the SER tubule is bridged to the inner surface of the axolemma (axol) by numerous matrix filaments. At the bottom of Figure 2 a portion of another axon is seen. Two SER tubules are present, one at the upper right which apparently terminates to the left, and another tubule which is sectioned tangentially and appears as a dense region under the number 2 at the lower left. The upper SER profile shows a dilated region where the area of the cisternum (cist) is greatly increased. A neurofilament ( $n f$ ) can be seen at the left which is extensively bridged to the SER (lower left arrow). Figure 3 shows part of an SER profile with numerous lateral filaments (arrows) extending from its surface; a microtubule $(m t)$ is shown at the bottom. The upper matrix filaments appear to bridge the SER to the axolemma at the top. Figure 4 shows a portion of an axon with two interconnected neurofilaments ( $n f)$; the lower neurofilament is bridged at two points to the SER tubule. In Figure 5 , a single neurofilament ( $n f$ ) is seen at the top of the axon in association with a tubule of SER which terminates blindly at the right (arrow). Note that the neurofilament appears to be connected to the axolemma at the top by numerous matrix filaments and that the cisternal space $(x)$ of the SER is clearly seen. Two microtubules $(m t)$ are evident, and the upper microtubule may be bridged to the SER tubule. Magnifications: Figure 2, $\times 352,000$; Figures 3 and 5, $\times 276,000$; Figure 4, $\times 324,000$. Bars $=0.05 \mu \mathrm{m}$.

neurofilaments to the left of the SER from section to section, it can be seen that the middle neurofilament changes its position in space. Generally, neurofilaments follow a more wandering path through space than do the microtubules, which tend to be relatively straight (Fig. 13a). Not all axon cross-sections show profiles of SER; in the middle axon in Figure 14 ( $a$ to $o$ ), there is no evidence of SER profiles in the sections, nor do neurofilaments appear to be present. This indicates that both organelles may be discontinuous along the length of axons. Although SER and microtubules are seen in the bottom axon of this series, there is no evidence of neurofilaments. In examining many series of 10 to 20 serial sections of olfactory axons, neurofilaments are often seen to be absent from given axons, or sometimes are discontinuous, whereas only a few axons are seen to be devoid of SER profiles in the series. Rarely are axons ever found to be devoid of microtubule profiles.

Although a single profile of SER is typically seen in an axon, it is not uncommon to see an axon with two or more profiles (Figs. 1, 2, and 11). Serial sectioning and computer-assisted reconstruction of such axons indicate that SER tubules may branch and anastomose, often in a complex manner, as shown in Figure 15. In the first section of this series, a single profile of SER is seen (Fig. 15a, open arrowhead). In the next section (Fig. 15b, open arrowheads), two 
profiles are seen to be connected by a tangentially sectioned membrane. Thereafter, two distinct profiles appear, followed by three profiles in Figure $15, d$ to $i$. In Figure $15, f$ and $g$, one of the three profiles shows dense material in the SER cisternum ( $X$ in Fig. $15, f$ and $g$ ). Further along, the three profiles are replaced by only two in Figure 15i, and then the third SER profile reappears with a large dense mass in its cisternal space (Fig. 15, $k$ to $m$ ), which disappears in Figure $15 n$, along with one of the SER profiles. Finally, in the 15th and last section in this series, only two profiles of SER are seen, one having a dumbbell-shaped outline (top profile in Fig. 150 ). Obviously, the SER in this stretch of axon is in the form of a tubular network of sorts, and only by studying serial sections can one begin to appreciate the 3-D nature of the network.

In order to better visualize the distribution in space of the SER profiles in the series shown in Figure 15, the profiles were digitized and subjected to computer-assisted reconstruction. Stereoscopic pairs of the profiles were plotted, and these are shown in Figure 16, $a$ and $b$, and Figure 16, $c$ and $d$; the stippled regions of the profiles in Figure 16, $a$ and $b$, represent tangential sections through SER membranes. The two small $x$ symbols in sections 6 and 7 (counting from left to right) in Figure 16a represent the cisternal spaces where dense material was seen in Figure $15, f$ and $g$, and the large and dense cisternal masses seen in this series are outlined in reconstructed profiles representing sections 11,12 , and 13 . The reconstructions in Figure 16, $a$ and $b$, were both plotted with an $X$-axis rotation of $45^{\circ}$, and the $Y$-axes were rotated at 70 and $75^{\circ}$ to provide the $5^{\circ}$ difference required for a stereoscopic effect. Figure 16, $c$ and $d$, show these same digitized profiles in a different perspective; again, stereoscopic images were plotted, but in this case the $X$-axis was 0 , the $Z$-axis rotation was $90^{\circ}$ in both cases, and the $Y$-axes differed by $5^{\circ}$ in their rotation $\left(60\right.$ and $\left.65^{\circ}\right)$. Obviously, stereoscopic views of reconstructed SER profiles are valuable in providing a better understanding of the 3-D arrangement of the SER seen in Figure 15. Figure 16, e and $f$, provides a stereoscopic view of SER profiles in a set of 17 serial sections, where the SER follows a particularly wandering path through space and also varies greatly in the dimensions of its cisternal space from one section to the next. In section 4 in this series (beginning with that section farthest from the viewer), there are two profiles, indicating branching of the SER, but by section 5 the two tubular branches have apparently fused, since only a single profile is seen in the series from this point in space toward the viewer. Note that the SER cisternum is greatly expanded as profiles 14 to 17 appear in space closer to the viewer. The stereoscopic pairs were generated by rotating around the $Y$-axis at $25^{\circ}$ (Fig. 16e) and $20^{\circ}$ (Fig. 16f).

It was determined in preliminary studies that SER tubules of olfactory axons were positively stained when secondarily fixed in osmium tetroxide ferricyanide, a method used by others to identify SER in a variety of cell types, including sarcoplasmic reticulum (Forbes et al., 1977; Schnepf et al., 1982; Walz, 1982a). Since the
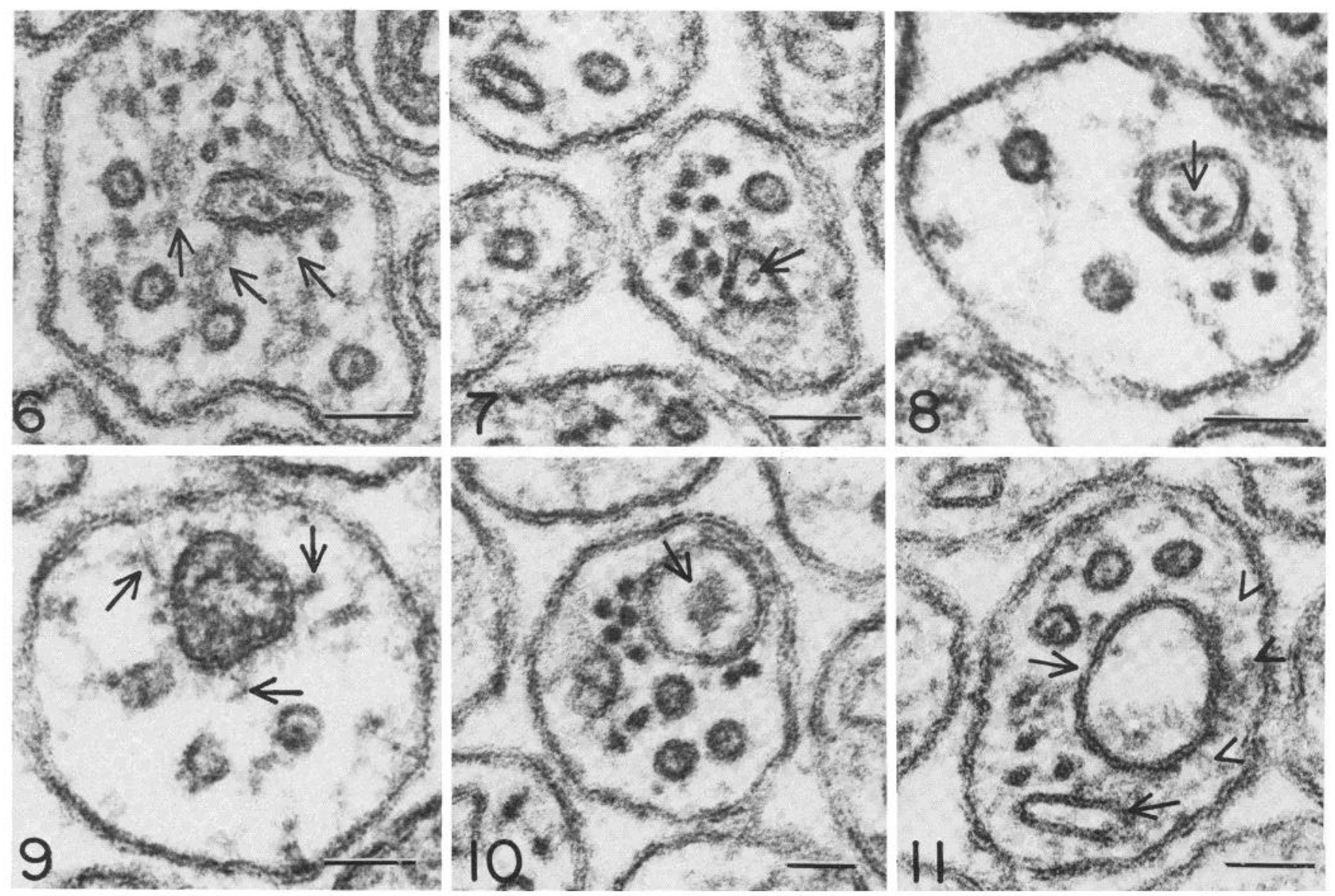

Figures 6 to 11. Cross-sections of axons showing dense material in the cisternal spaces of SER. Figure 6 shows a small mass of material in the cisternum; filaments are seen extending from the surface of the SER. Figure 7 also shows a small, compact mass (arrow) in the cisternum of an SER tubule. Figure 8 shows a somewhat larger mass (arrow) in an SER lumen, but the mass is rather amorphous. Figure 9 shows an SER tubule in which the cisternum is filled with dense material; filaments can be seen (arrows) extending from the surface of the tubule. The SER tubule in Figure 10 shows a mass of material (arrow) in its cisternal space; note the close association between neurofilaments and the SER tubule. Figure 11 shows two profiles of SER (arrows), with the larger SER tubule containing loosely distributed amorphous material. Matrix filaments are associated with the surface of the larger tubule (open arrowheads at the right). Magnifications: Figures 6, 7, and 11, × 237,000; Figure 8, $\times 276,000$; Figure 9, $\times 243,000$; Figure $10, \times 265,000$. Figure 10 was provided by Dr. Daryl Hartter. Bars $=0.05 \mu \mathrm{m}$. 

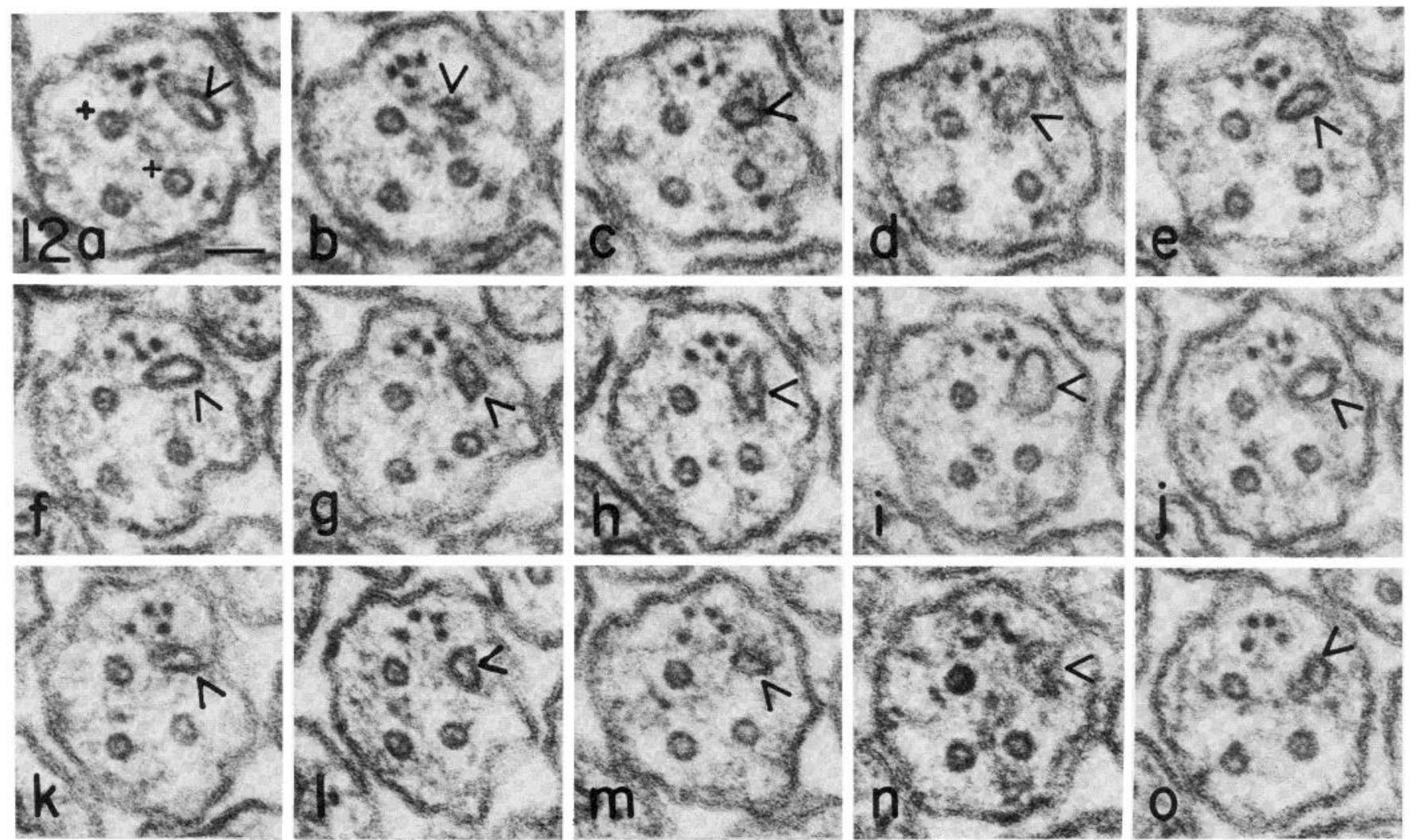

Figure 12. Axon shown in 15 serial cross-sections ( $a$ to o). The SER profile is shown by the open arrowheads; note that the profile is closely associated with a cluster of four neurofilaments. In digitizing this series for computer-assisted reconstruction, the two microtubules shown by the + symbol were used as the two internal reference points, since initial inspection of the series indicated that their relative positions in space remained the same throughout (i.e., they appeared straight). Note the changes in shape of the SER profile from micrograph to micrograph. The reconstruction of this stretch of axon is shown in Figure $13 a$ and represents a total estimated length of slightly over $1 \mu \mathrm{m}$. Magnification $\times 146,000 ;$ bar $=0.05 \mu \mathrm{m}$.

Ca-sequestering ability of sarcoplasmic reticulum is well known, and since Walz $(1979,1982 a)$ amply demonstrated the ability of SER in insect photoreceptor cells to sequester $\mathrm{Ca}$, his methods were employed in attempting to demonstrate the active pumping of $\mathrm{Ca}$ by SER of frog olfactory axons. As shown in Figures 17 to 22, the results were positive. Pieces of olfactory nerve were briefly incubated in medium containing saponin to permeabilize axons and then were incubated in the presence of ATP, oxalate, and micromolar levels of $\mathrm{Ca}$; material thereafter fixed, sectioned, and examined with the electron microscope showed the electron-dense calcium oxalate specifically associated with SER profiles.

A longitudinal profile of SER from an axon in an experimental nerve piece is shown in Figure 17, where the reaction product (calcium oxalate) is seen to be rather uniformly distributed in the cisternal space. If samples were incubated in medium devoid of ATP ( $5 \mathrm{~mm}$ ), little if any product was seen to be associated with SER profiles (Fig. 20). Likewise, very little product was seen if nerve pieces were permeabilized and then incubated in medium containing apyrase, to inhibit ATP, or in medium devoid of oxalate (Figs. 21 and 22). In cross-sections of experimental axons, the delicate but very obvious reaction product was usually specifically associated with SER profiles (Figs. 18 and 19), where it appeared either within the cisternal space or along the SER membrane. Mitochondria rarely showed evidence of calcium oxalate, indicating that at the low concentration of $\mathrm{Ca}$ used the organelles sequestered little if any of the ion. The specificity of the reaction product was often striking in unstained cross-sections of axons, where electron-dense material was associated with almost all of the SER profiles (Figs. 18 and 19). With the cytochemical methods used, no differences were noted between the distribution of reaction product in proximal or distal portions of axons in the olfactory nerve, with reference to their perikarya in the olfactory epithelium of the frog.

\section{Discussion}

Even though it is possible that in some neurons the SER forms a continuous uninterrupted network from the perikaryon to its axonal terminals, this is clearly not the case in frog olfactory neurons. In olfactory axons the SER is continuous over great distances, but it is nonetheless a discontinuous system, as shown by studying and reconstructing serial sections in 3-D space. The longitudinal continuity of SER was earlier questioned on the basis of studies of pike olfactory axons, where it was reported that SER profiles could not be found in $14 \%$ of the axon cross-sections examined (Kreutzberg and Gross, 1977). Unless serial sections were studied, however, it might be easy to overlook SER profiles in such small axons in a given section when the membrane was cut tangentially, especially at relatively low magnifications.

The possibility that SER serves as a vector or is a conduit for fast axoplasmic transport is an interesting hypothesis that has not been confirmed in recent studies. The association of rapidly transported protein with SER profiles, as reported by Droz et al. (1975), is difficult to explain. The rapid accumulation of "vesiculotubular" elements in axons on the proximal side of a cold block, as reported by Tsukita and Ishikawa $(1979,1980)$, is not sufficient to implicate SER as a rapidly transported membrane compartment. Considering that most axons contain, in addition to SER membranes, various kinds of vesicles and vacuoles, it would be difficult to identify SER membranes with certainty unless a characteristic property could be 
demonstrated, such as the cytochemical demonstration of specific enzymes (Teichberg and Holtzman, 1973) or the ability to sequester Ca.

Ellisman and Lindsey (1983) used a focal chilling method to block axoplasmic transport at closely adjacent sites along saphenous nerves of mice. By studying the accumulation of material on either side of such sites, as well as in the warmed region separating the sites, ultrastructural evidence was obtained showing that SER is not rapidly transported through the axon, indicating that the membrane system probably is not a fast-moving conveyer belt translocating attached components by membrane flow. The findings were dis- cussed as they related to the transport of membranous elements within axons; whatever the role of SER in axoplasmic transport, the recent evidence indicates that its involvement is limited to anterograde movement and that SER membranes are distinct from the spherical or slightly elongated membranous elements associated with retrograde transport (LaVail et al., 1980; Rambourg and Droz, 1980).

In examining serial sections and computer reconstructions of frog olfactory axons, there was little evidence of vesicles associated with the SER, as if undergoing fusion. In fact, normally, these axons are devoid of vesicles and vacuoles. It is thus unlikely that the shuttling


Figure 13. Computer-assisted reconstructions of the axon shown in serial sections in Figure 12 (a to o). a shows digitized profiles plotted for all of the major formed elements of the axon (axulemnla, microtubules, meurofilaments, and SER), along with, the SER profiles of an adjacent axon, at the bottom. Although the SER profiles in the completely reconstructed axon follow a relatively straight path, those in the neighboring axon follow a wandering course through space, even though the center-to-center distance between the two axons remained identical over the course of the 15 sections. Note that the neurofilament at the lower left follows an oblique path, relative to the longitudinal axis of the axon. This reconstruction was plotted at a $Y$-axis setting of $65^{\circ}$ (i.e., each section was rotated around its vertical axis by $65^{\circ}$ ). To show more clearly the 3-D course of the SER, two plots were made in which only the SER and axolemmal profiles were displayed, with the two differing by $5^{\circ}$ around the $X$-axis (the $Y$-axis remained at $65^{\circ}$ ) to provide stereoscopic pairs. These are shown in $b$ and $c$. 

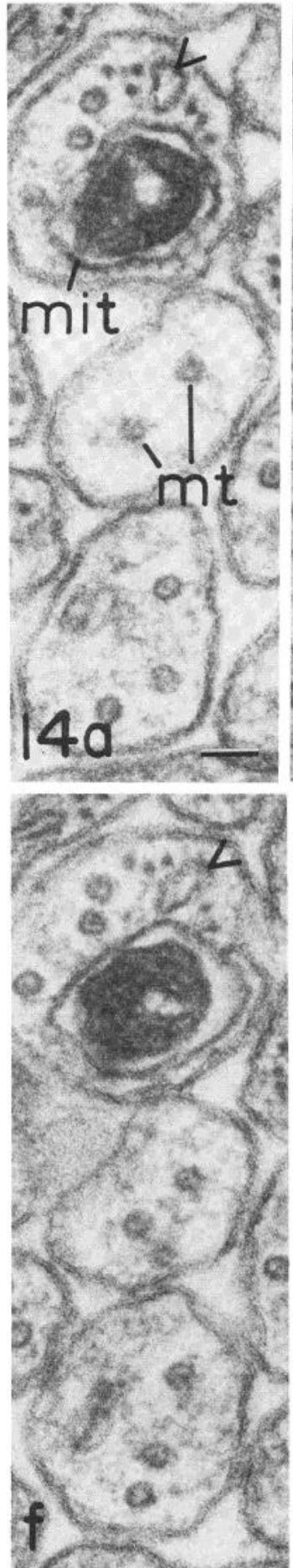
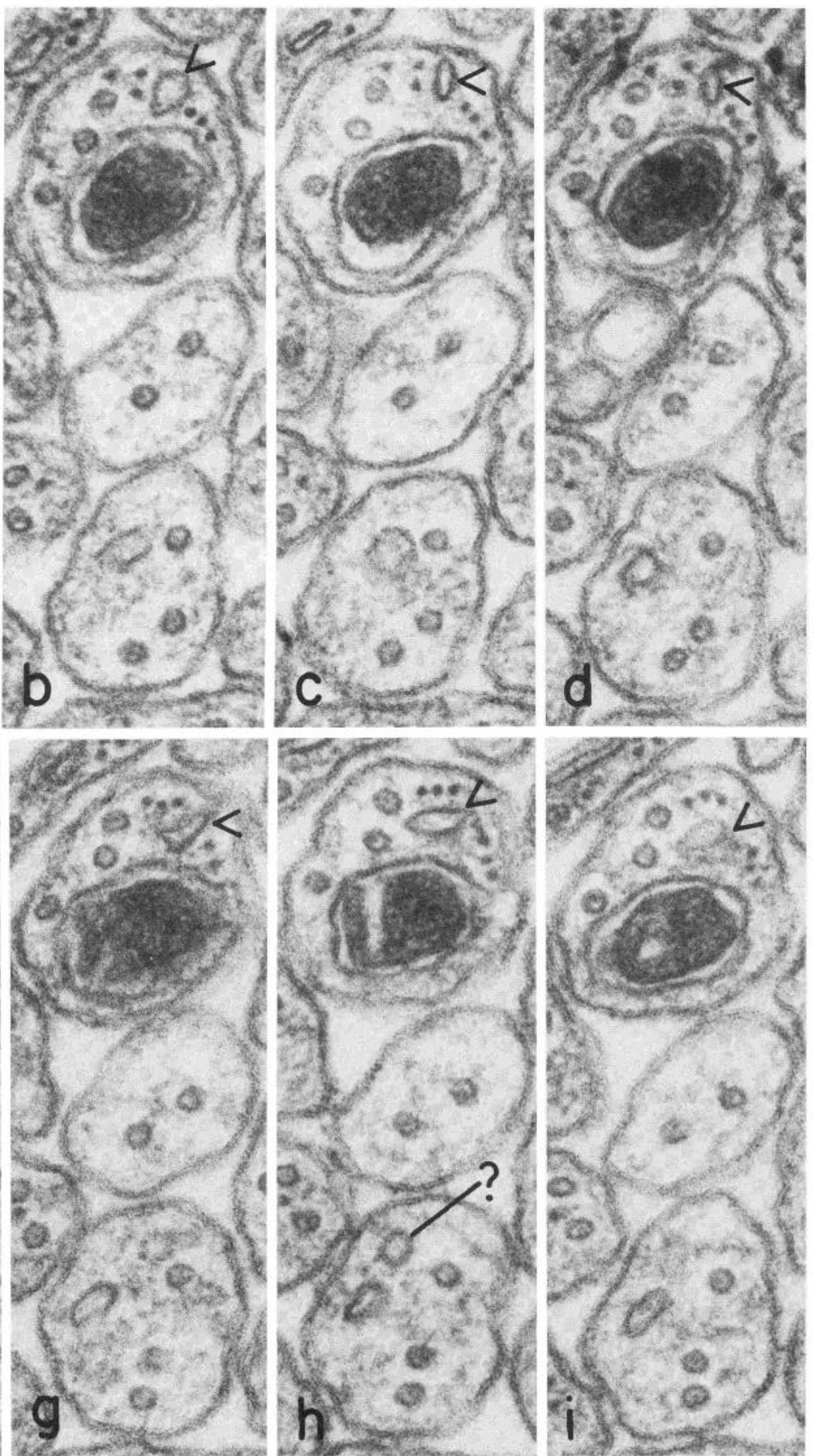
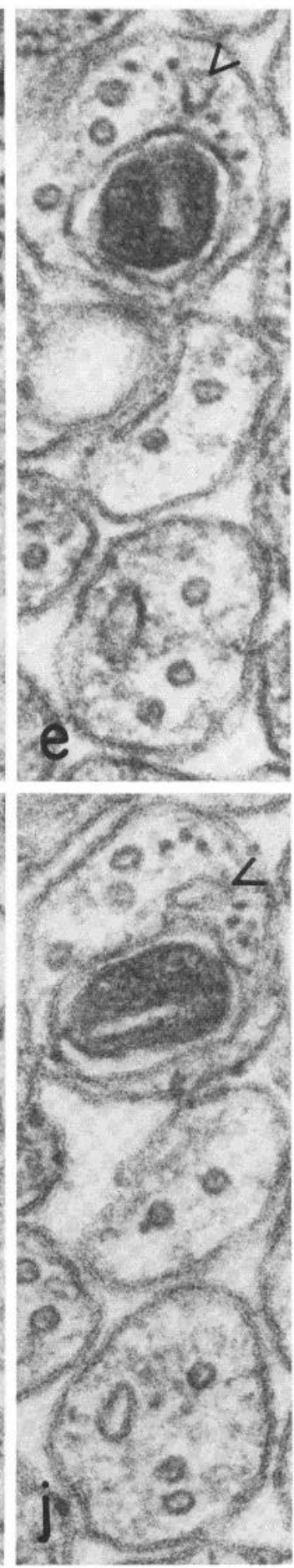

Figure 14. Three olfactory axons are shown in 15 serial cross-sections ( $a$ to o). A mitochondrial (mit) profile is seen in the axon at the top. The single SER tubule is indicated by the open arrowhead, and the tubule is associated with six neurofilaments. In contrast, the three microtubules form a domain of their own to the left which is characterized by a relatively clear area around the microtubule profiles. Note that the neurofilament profiles tend to wander in space from section to section. For example, follow the path of the lower neurofilament profile of the three to the left of the SER profile in a. The middle axon in this series shows no evidence of an SER tubule or neurofilament profiles, containing only two microtubules (mt). The lower axon is also devoid of neurofilament profiles, although an SER tubule is present. The membrane profile in $h$ may be a vesicle, although vesicles are rarely seen in frog olfactory axons. Magnification $\times 135,000 ;$ bar $=0.05 \mu \mathrm{m}$.

of vesicles pinched off the SER occurs in olfactory axons, as suggested by Tsukita and Ishikawa (1976). The occurrence of various forms of electron-dense material in the cisternae of the SER of olfactory axons does, however, provide evidence that tubules of SER may serve as conduits for certain components (see Teichberg and Holtzman, 1973; Alonso and Assenmacher, 1979). Whether such material is actively or passively translocated is unknown. If the SER tubule varied its dimensions in a wave-like manner, either as a result of force generation intrinsic to the membrane or as a result of extrinsic forces imposing shape changes on the tubule, cisternal 

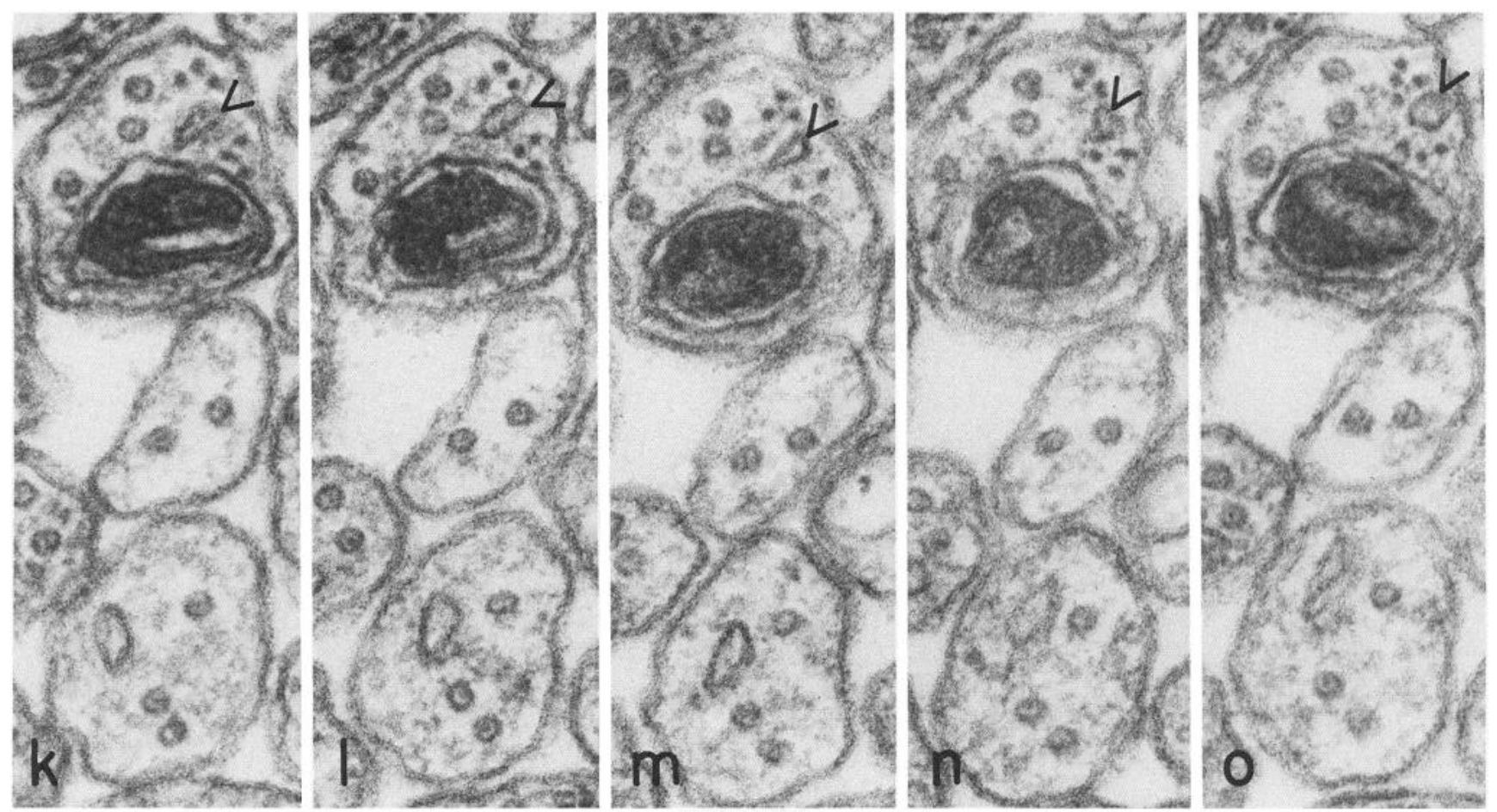

Figure 14. $k-0$
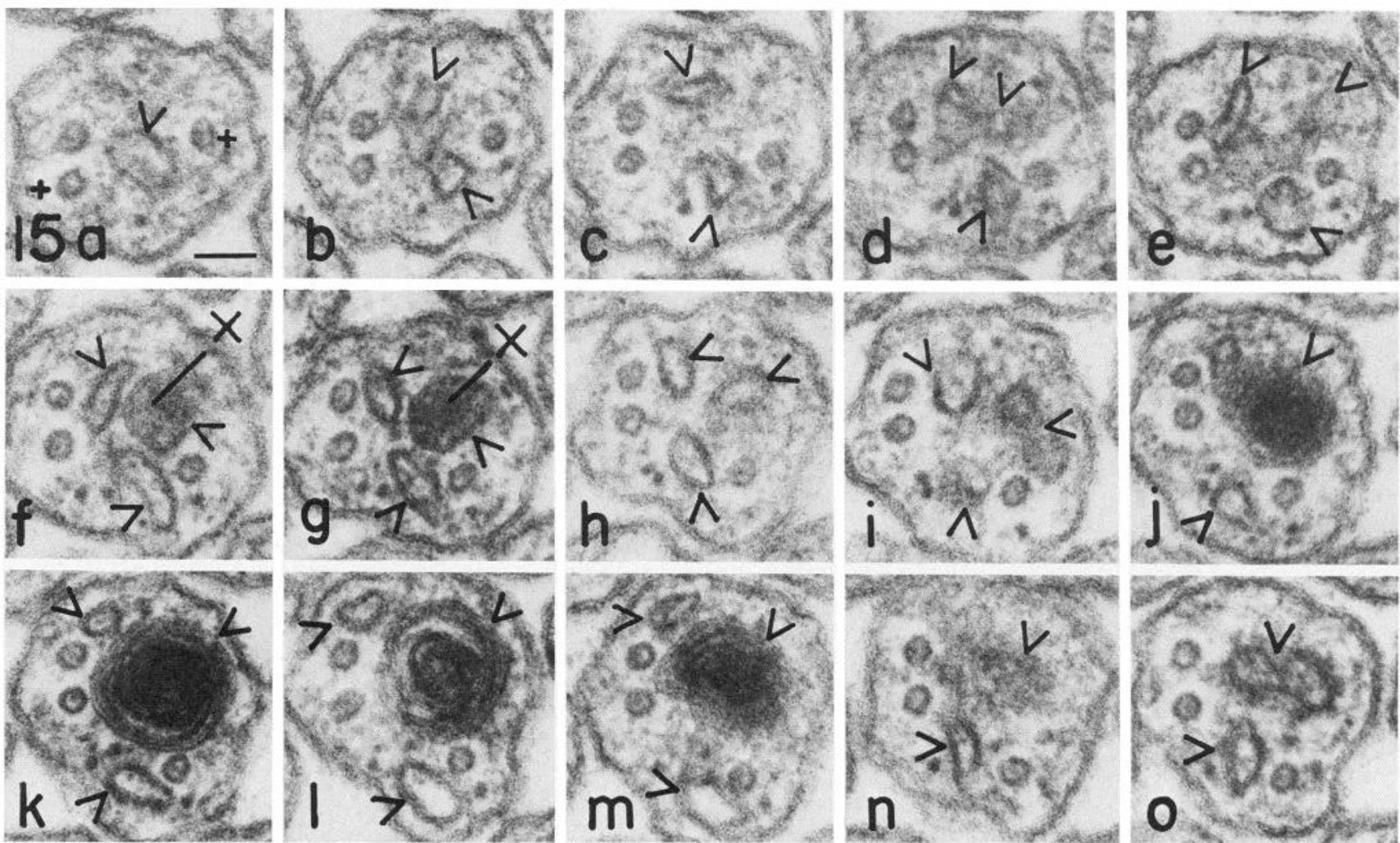

Figure 15. Axon shown in 15 serial cross-sections (a to o). Components of this axon were digitized and subjected to reconstruction, with two of the three microtubules used as internal reference points (+ symbols). The SER in the first cross-section appears as a single profile (open arrowhead). In subsequent sections, two or three profiles are seen, all shown by the open arrowheads, indicating that the tubule had undergone branching and anastomosing. One of the three SER profiles in $f$ and $g$ shows dense luminal material $(x)$, which is no longer seen in $h$. In $i$, however, dense material reappears and remains through $n$, where the SER membrane around the luminal material is sectioned tangentially. The SER in this series is reconstructed in Figure 16 , a to $d$. Magnification $\times 146,000 ;$ bar $=0.05 \mu \mathrm{m}$. 

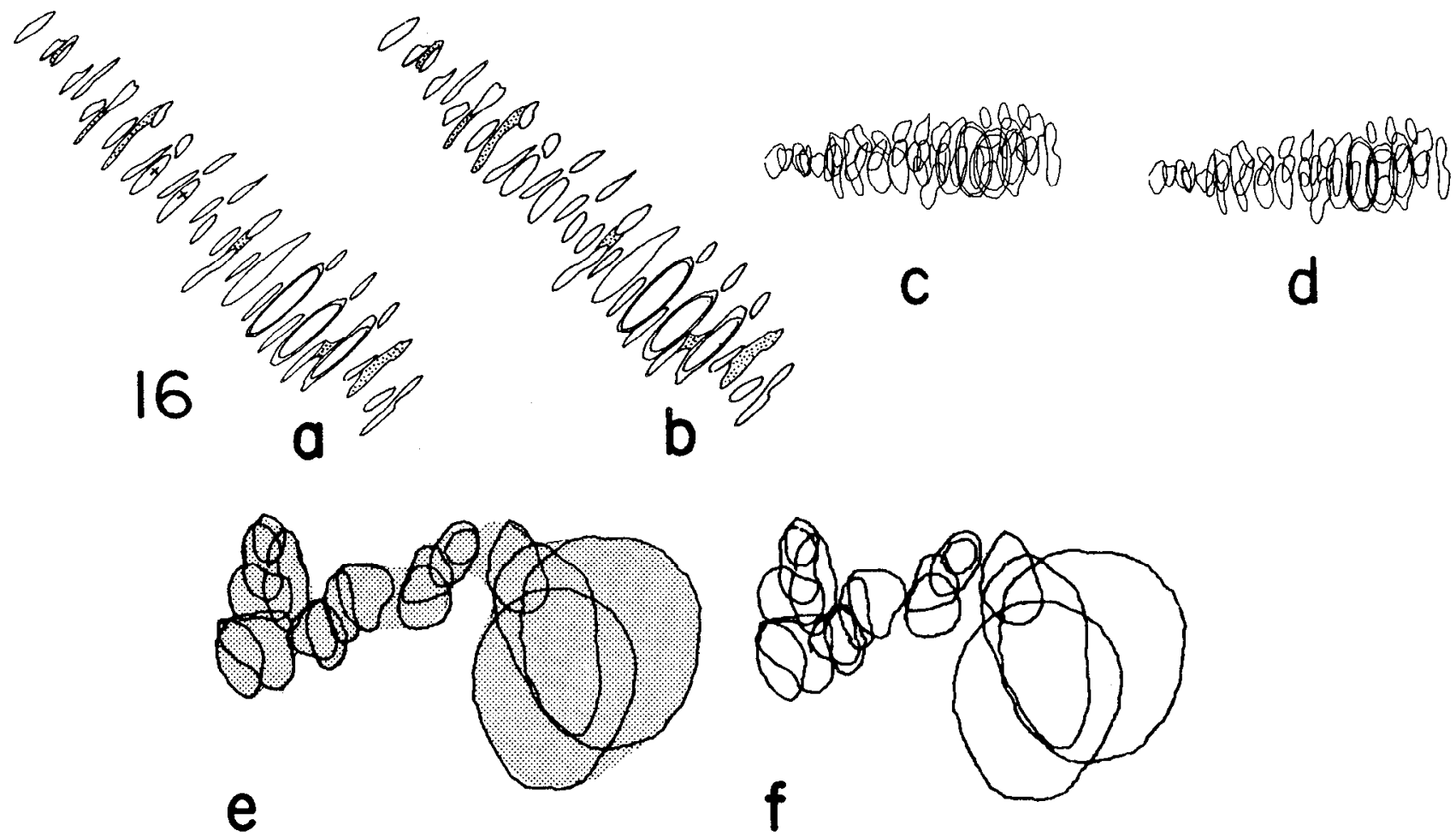

Figure 16. The SER profiles in the axon shown in Figure 15 are reconstructed in stereoscopic pairs here ( $a$ to $d$ ). In $a$ and $b$, the SER profiles are rotated $45^{\circ}$ around the $X$-axis and 70 and $75^{\circ}$ around the $Y$-axis. Tangential or grazing sections through membranes are shown by stippling, and the SER profiles corresponding to Figure 15, $f$ and $g$, where dense material appears in the cisternum, are shown by the letters $x$ in $a$. The large dense masses seen in the cisternal space of the SER profile in Figure $15, k$ to $m$, are shown in profile view. The stereoscopic pairs shown here in $c$ and $d$ provide a slightly different perspective of the SER profiles seen in Figure 15 ( $a$ to 0 ). The best stereoscopic effect is obtained with a magnifying viewer, and when viewed in true 3-D in this manner, one appreciates the complex path followed by this system of SER tubules. in $c$ and $d$, the $X$-axis was 0 , the $Y$-axis was 60 and $65^{\circ}$, and the $Z$-axis was $90^{\circ}$. e and $f$ show a stereoscopic reconstruction from 17 serial cross-sections of SER, where the tubule is seen to follow a highly tortuous path with a great increase in cisternal area (volume) in the SER profiles closest to the eye of the viewer. Also, there is one apparent point of branching and anastomosing in this series (see the text for details). $e$ is overlaid with a gray dot pattern to enhance the 3-D effect when the images are visually fuscd.

material could be translocated. The SER tubules indeed often follow tortuous paths and show great variation in the volume of their cisternal spaces, but the signilicance of this is unclear.

There has been much interest in the filamentous components interlinking neurofilaments, microtubules, and membranous elements of axons (Metuzals, 1969; Burton and Fernandez, 1973; Ellisman and Porter, 1980; Hodge and Adelman, 1980; Hirokawa, 1982), and there can be no doubt that these bridging macromolecules are real and that they play important but unknown roles in the functional integrity of neurons. In olfactory axons, the SER was often seen to be bridged to neurofilaments and, to a lesser extent, to microtubules. Although the function of neurofilaments is unknown, they and microtubules appear to be among the slowest-moving components of the axoplasm (Brady and Lasek, 1982), and it is likely that at least one function of these relatively stationary linear elements is to provide the axon with a stable internal skeleton. If this is so, then it is curious that such varied numbers of neurofilaments are seen in olfactory axons of comparable diamcter, and that some axons show no neurofilaments at all.

The frequency with which tubules of SER were associated with and bridged to neurofilaments in olfactory axons suggests an often shared axoplasmic domain, and the bridging between these organelles could be cited as structural evidence against fast translocation of SER tubules. Kreutzberg and Gross (1977) recognized two axoplasmic domains in axons of pike olfactory axons. The authors noted that microtubules were not uniformly distributed but show ". . a regional preference that is always in opposition to the neurofilament clusters seen often in axonal cross sections" (Kreutzberg and Gross, 1977, p. 452). Although not mentioned, their micrographs show many axons in which a close association exists between profiles of SER and groups of neurofilaments. There is more apparent variation in frog olfactory axons than in those of the pike, and in frog axons the microtubule domain is not always in opposition to the neurofilament domain. This may be related to the great variation in neurofilament number in frog axons. Obviously, consideration of the domains occupied by linear organelles of the axoplasm is of great importance in attempting to understand axoplasmic transport.

One of the most prominent domains seen in frog olfactory axons is that occupied by the microtubules; aside from the microtubules themselves, the most conspicuous feature of this domain is the frequent absence of filamentous matrix material, providing a lucent or clear zone around the microtubules. Such a zone has been often seen around profiles of microtubules, and it is discussed and referred to as the "exclusion zone" by Dustin (1978). The significance of this zone is unclear. However, there is growing evidence that translocation of molecules and even organelles can occur along the surfaces of microtubules (Schliwa, 1984; Koonce and Schliwa, 1985; Schnapp et al., 1985), and that microtubules play a role in fast axoplasmic transport. The general acceptance of the involvement of microtubules in fast transport is based, in part, on dozens of studies showing that antimicrotubule drugs (e.g., colchicine) inhibit translocation (first shown by Dahlstrom, 1968; see Heslop, 1975, for review). Furthermore, several studies have shown that microtubules appear well before neurofilaments in neurons of newborn mammals (Smith, 1973; Pachter and Liem, 1981), and that fast axoplasmic transport occurs in developing neurons (Specht, 1977), even in the prcsumed absence of neurofilaments. Recently, $\beta, \beta^{\prime}$-iminodipropionitrile was 

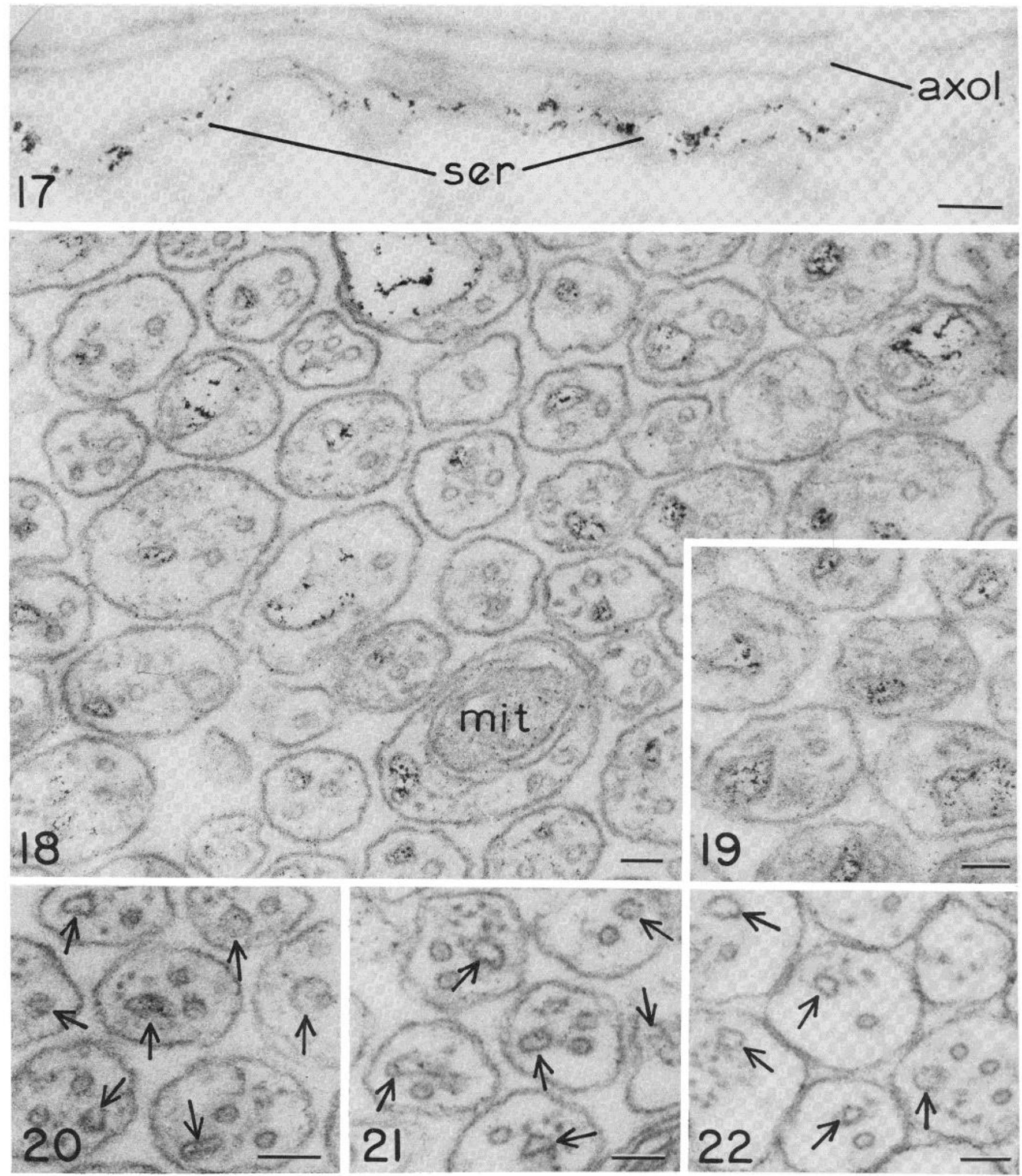

Figures 17 to 22. These micrographs show the results obtained with a cytochemical test demonstrating the ability of olfactory SER tubules to sequester exogenous $\mathrm{Ca}$ in the presence of ATP. The reaction product was electron-dense calcium oxalate. Experimental material is shown in Figures 17 to 19 . Figure 17 shows reaction product in an SER tubule seen in longitudinal profile; dense product is clearly restricted to the SER. Experimental axons are shown in cross-section in Figures 18 and 19, where almost all profiles of SER contain reaction product. Very little, if any, reaction product is seen in material incubated without ATP (Fig. 20), in medium containing apyrase to inhibit ATP (Fig. 21), or in medium devoid of oxalate (Fig. 22). See the text for further details. Magnifications: Figure 17, × 264,000; Figure 18, × 146,000; Figure 19, $\times 162,000$; Figure 20, $\times 216,000$; Figures 21 and 22, $\times 184,000$. Bar $=0.05 \mu \mathrm{m}$. 




Figure 23. This cartoon diagram conveys the two functions proposed for the SER of frog olfactory axons. The primary function is to play a role in maintaining functionally appropriate levels of Ca in the axuplasm by sequestering the ion using an ATP-dependent mechanism associated with the membrane (a pump, if you will). A secondary function is that of providing a tubular compartment through which components (symbolized by the apple) might be translocated. Whether such translocation occurs in a passive or active manner, symbolized by the wagon attached to the question mark, is unknown.

found to induce the redistribution of microtubules and neurofilaments of axons into separate and distinct groups (Papasozomenos et al., 1981), and autoradiography was used to show that labeled proteins translocated at the fast rate were associated with the group of microtubules and not with that of neurofilaments (Papasozomenos et al., 1982; Griffin et al., 1983). Collectively, such findings indicate that microtubules are somehow involved in fast axoplasmic transport probably by serving as guideways and attachment sites for some macromolecule providing the motive force for translocation (Burton and Fernandez, 1973; Schliwa, 1984).

Since it is known that fast and slow axoplasmic transport occur in olfactory axons (Gross and Beidler, 1973; Gross and Kreutzberg, 1978; Weiss et al., 1978; our own unpublished studies with frog olfactory nerve), the obvious channel through which fast transport occurs is that surrounding microtubules, or through the exclusion zone. In this regard, of the many hypotheses attempting to explain the mechanism of fast axoplasmic transport, one stands out for its elaborate concern with the axoplasmic channels through which movement is likely to occur. This is the "microstream" hypothesis originally set forth by Gross (1975) and recently updated by Weiss and Gross (1982). Although this theory has little to say about specific macromulecules that could bridge a component to a microtubule and undergo a conformational change to move the component in space along the microtubule, the theory deals at length with the biophysical nature of the axoplasm and the low viscosity channels through which fast transport surely occurs. In the case of olfactory axons, one is quickly struck with the likelihood that the domain around the neurofilaments and SER, often enmeshed in a matrix network of sorts, was in a gel state in life, and that the domain of exclusion around the microtubules was in a low viscosity sol state. This would be much in keeping with some of the precepts on which the microstream hypothesis is based (Gross and Weiss, 1982).

The available evidence indicates that one of the main functions of axonal SER is to maintain appropriate physiological levels of $\mathrm{Ca}$ in the axoplasm, and that mitochondria probably provide a backup sink, but only at $\mathrm{Ca}$ levels well above the physiological range (Blaustein et al., 1980; Brinley, 1980; McGraw et al., 1980; Requena, 1983; Zucker and Stockbridge, 1983), which in squid axons was measured to be 30 to $50 \mathrm{nM}$ (Brinley, 1980). Intracellular Ca participates in many cellular events, usually by binding with and altering the properties of a specific binding protein; in neurons, investigators have focused on the role of $\mathrm{Ca}$ in initiating or sustaining axoplasmic transport (Hammerschlag, 1980; Ellisman, 1982; Kanje et al., 1982;
Ochs, 1982), triggering neurotransmitter release (Blaustein el al., 1980 ), degrading neurofilaments in the axon terminals (or after injury) by activation of proteases (Schlaepfer and Bunge, 1973; Schlaepfer, 1974; Gilbert, 1975; Lasek and Hoffman, 1976; Schlaepfer et al., 1981; Pant et al., 1982), and regulating the assembly-disassembly of microtubules (Yamauchi and Гujisawa, 1982; Yamamoto et al., 1983). In the case of frog olfactory axons, SER clearly has the capacity to actively sequester $\mathrm{Ca}$, and it is likely that SER is mainly responsible for maintaining functionally appropriate levels of axoplasmic $\mathrm{Ca}$. Furthermore, it is a reasonable assumption that $\mathrm{Ca}$ plays a role in fast axoplasmic transport in olfactory axons, and that its presence at some critical level also provides for the gel-sol states required to maintain open channels through which components can be translocated. In regard to regulating levels of axoplasmic $\mathrm{Ca}$, a means must exist to trigger the release of sequestered $\mathrm{Ca}$ from the $\mathrm{SER}$ in a controlled manner to adjust the $\mathrm{Ca}$ cquilibrium in the axoplasmic matrix.

Although the primary function of SER in olfactory axons is undoubtedly that of $\mathrm{Ca}$ regulation, as in other axons, the frequent appearance of dense material in the cisternal space suggests a secondary function for these elongate compartments. Material could aiso be translocated within the lumen of the SER, but probably in a passive manner; one must remember, however, that the SER tubule is not a static structure with a constant shape, and that it undoubtedly undergoes limited fusion and fission as it slowly moves from the perikayon into the axon proper on its journey toward the terminals (see Hammerschlag and Stone, 1982). The cartoon shown in Figure 23 summarizes the proposed functions of SER in olfactory axons, based on our studies to date. The major function is to pump and sequester $\mathrm{Ca}$, but a secondary function is that of providing a tubular compartment through which material, symbolized by the apple, could be translocated. This probably occurs in a passive manner, but this remains uncertain, as symbolized by the wagon to which a question mark is attached.

As in other vertebrates, there is good evidence that olfactory neurons of the frog undergo slow replacement (Graziadei and Metcalf, 1971; Graziadei, 1973; Moulton, 1974). Thus, some of the axons in the adult olfactory nerve represent newly established extensions from perikarya in the olfactory epithelium. Although we have tentative criteria for distinguishing such "new" axons, the observations made in the present paper mainly focus on what appear to be typical, well established axons. Future research will deal more specifically without growing axons from newly differentiated neurons.

\section{References}

Alonso, G., and I. Assenmacher (1979) The smooth endoplasmic reticulum in neurohypophysial axons of the rat: Possible involvement in transport, storage and release of neurosecretory material. Cell Tissue Res. 199: 415429.

Blaustein, M. P., R. W. Ratzlaff, and E. S. Schweitzer (1980) Control of intracellular calcium in presynaptic nerve terminals. Fed. Proc. 39: 27902795

Brady, S. T., and R. J. Lasek (1982) The slow components of axona transport: Movements, compositions and organization. In Axoplasmic Transport, D. G. Weiss, ed., pp. 206-217, Springer-Verlag, Berlin.

Brinley, F. J. (1980) Regulation of intracellular calcium in squid axons. Fed. Proc. 39: 2778-2782.

Burton, P. R., and H. L. Fernandez (1973) Delineation by lanthanum staining of filamentous elements associated with the surfaces of axonal microtubules. J. Cell Sci. 12: 567-583.

Burton, P. R. (1984) Luminal material in microtubules of frog olfactory axons: structure and distribution. J. Cell Biol. 99: 520-528.

Burton, P. R. (1985) Computer-assisted three-dimensional reconstruction of the ultrastructure of the frog olfactory axon. Norelco Reporter 32: 1-10.

Chan, S. Y., S. Ochs, and R. $\wedge$. Jersild (1984) Localization of calcium in nerve fibers. J. Neurobiol. 15: 89-108.

Dahlstrom, A. (1968) Effect of colchicine on transport of amine storage granules in sympathetic nerves of rat. Eur. J. Pharmacol. 5: 111-113.

Droz, B., A. Rambourg, and H. L. Koenig (1975) The smooth endoplasmic 
reticulum: Structure and role in the renewal of axonal membrane and synaptic vesicles by fast axonal transport. Brain Res. 93: 1-13.

Duce, I. R., and P. Keen (1978) Can neuronal smooth endoplasmic reticulum function as a calcium reservoir? Neuroscience 3: 837-848.

Ducros, C. (1974) Ultrastructural study of the organization of axonal agranular reticulum in Octopus nerve. J. Neurocytol. 3: 513-523.

Dustin, P. (1978) Microtubules, pp. 33-34, Springer-Verlag, Berlin.

Ellisman, M. H. (1982) A hypothesis for rapid axoplasmic transport based upon focal interactions between axonal membrane systems and the microtrabecular crossbridges of the axoplasmic matrix. In Axoplasmic Transport, D. G. Weiss, ed., pp. 390-396, Springer-Verlag, Berlin.

Ellisman, M. H., and J. D. Lindsey (1983) The axoplasmic reticulum within myelinated axons is not transported rapidly. J. Neurocytol. 12: 393-411.

Ellisman, M. H., and K. R. Porter (1980) Microtrabecular structure of the axoplasmic matrix: Visualization of cross-linking structures and their distribution. J. Cell Biol. 87: 464-479.

Fifkova, E., J. A. Markham, and R. J. Delay (1983) Calcium in the spine apparatus of dendritic spines in the dentate molecular layer. Brain Res. 266: 163-168.

Forbes, M. S., B. A. Plantholt, and N. Sperelakis (1977) Cytochemical staining procedure sclcctivc for sarcotubular systems of muscle: Modifications and applications. J. Ultrastruct. Res. 60: 306-327.

Gilbert, D. S. (1975) Axoplasm chemical composition in Myxicola and solubility properties of ils slruclural proteins. J. Physiol. (Lond.) 253: 303-319.

Graziadei, P. P. C. (1973) Cell dynamics in the olfactory mucosa. Tissue Cell 5: 113-131.

Graziadei, P. P. C., and J. F. Metcalf (1971) Autoradiographic and ultrastructural observations on the frog's olfactory mucosa. Z. Zellforsch. 116: 305318.

Griffin, J. W., K. E. Fahnestock, D. L. Price, and P. N. Hoffman (1983) Microtubule-neurofilament segregation produced by $\beta, \beta^{\prime}$-iminodipropionitrile: Evidence for the association of fast axonal transport with microtubules. J. Neurosci. 3: 557-566.

Gross, G. W. (1975) The microstream concept of axoplasmic and dendritic transport. Adv. Neurol. 12: 283-296.

Gross, G. W., and L. M. Beidler (1973) Fast axonal transport in the c-fibers of the garfish olfactory nerve. J. Neurobiol. 4: 413-428.

Gross, G. W., and G. W. Kreutzberg (1978) Rapid axoplasmic transport in the olfactory nerve of the pike. I. Basic transport parameters for proteins and amino acids. Brain Res. 139: 65-76.

Gross, G. W., and D. G. Weiss (1982) Theoretical considerations on rapid transport in low viscosity axonal regions. In Axoplasmic Transport, D. G. Weiss, ed., pp. 330-341, Springer-Verlag, Berlin.

Hammerschlag, R. (1980) The role of calcium in the initiation of fast axonal transport. Fed. Proc. 39: 2809-2814.

Hammerschlag. R., and G. C. Stone (1982) Fast axonal transport as endomembrane flow. In Axoplasmic Transport, D. G. Weiss, ed., pp. 407-413, Springer-Verlag, Berlin.

Henkart, M. (1980) Identification and function of intraceliular calcium stores in axons and cell bodies of neurons. Fed. Proc. 39: 2783-2789.

Henkart, M. P., T. S. Reese, and F. J. Brinley (1978) Endoplasmic reticulum sequesters calcium in the squid giant axon. Science 202: 1300-1303.

Heslop, J. P. (1975) Axonal flow and fast transport in nerves. Adv. Comp. Physiol. Biochem. 6: 75-163.

Heumann, H. G., and E. Zebe (1967) Uber Feinbau und Funktionsweise der Fasern aus dem Hautmuskelschlauch des Regenwurms, Lumbricus terrestris. Z. Zellforsch. 78: 131-150.

Hirokawa, N. (1982) Cross-linker system between neurofilaments, microtubules, and membranous organelles in frog axons revealed by the quickfreeze, deep-etching method. J. Cell Biol. 94: 129-142.

Hodge, A. J., and W. J. Adelman (1980) The neuroplasmic network in Loligo and Hermissenda neurons. J. Ultrastruct. Res. 70: 220-241.

Kanje, M. A. Edstrom, and P. Fkstrom (198?) The role of $\mathrm{Ca}^{2+}$ in rapid axonal transport. In Axoplasmic Transport, D. G. Weiss, ed., pp. 394-300, Springer-Verlag, Berlin.

Kim, H., L. I. Binder, and J. L. Rosenbaum (1979) The periodic association of MAP2 with brain microtubules in vitro. J. Cell Biol. 80: 266-276.

Koonce, M. P., and M. Schliwa (1985) Bidirectional organelle transport can occur in cell processes that contain single microtubules. J. Cell Biol. 100. 322-326.

Kreutzberg, G. W., and G. W. Gross (1977) General morphology and axonal ultrastructure of the oltactory nerve of the pike, Esox lucius. Cell Tissue Res. 181: 443-457.

Lasek, R. J., and P. N. Hoffman (1976) The neuronal cytoskeleton, axonal transport and axonal growth. In Cell Motility, R. Goldman, T. Pollard, and
J. Rosenbaum, eds., pp. 1021-1049, Cold Spring Harbor Laboratory, New York.

LaVail, J. H., S. Rapisardi, and I. K. Sugino (1980) Evidence against the smooth endoplasmic reticulum as a continuous channel for the retrograde axonal transport of horseradish peroxidase. Brain Res. 191: 3-20.

Lieberman, A. R. (1971) Microtubule-associated smooth endoplasmic reticulum in the frog's brain. Z. Zellforsch. 116: 564-577.

McGraw, C. F., A. V. Somlyo, and M. P. Blaustein (1980) Probing for calcium at presynaptic nerve terminals. Fed. Proc. 39: 2796-2801.

Metuzals, J. (1969) Curifiguralion of a filamentous network of the axoplasm of the squid (Loligo pealii L.) giant nerve fiber. J. Cell Biol. 43: 480-505.

Moulton, D. G. (1974) Dynamics of cell populations in the olfactory epithelium. Ann. N. Y. Acad. Sci. 237: b2-61.

Ochs, S. (1982) Calcium and the mechanism of axoplasmic transport. Fed. Proc. 41: 2301-2306.

Pachter, J. S., and R. K. H. Liem (1981) Differential appearance of neurofilament triplet polypeptides in developing rat optic nerve. J. Cell Biol. 91: 86a.

Pant, H. C., P. E. Gallant, R. Gould, and H. Gainer (1982) Distribution of calcium-activated protease activity and endogenous substrates in the squid nervous system. J. Neurosci. 2: 1578-1587.

Papasozomenos, S., L. Autilio-Gambetti, and P. Gambetti (1981) Reorganization of axoplasmic organelles following $\beta, \beta^{\prime}$-iminodipropionitrile administration. J. Cell Biol. 91: 866-871.

Papasozomenos, S., M. Yoon, R. Crane, L. Autilio-Gambetti, and P. Gambetti (1982) Redistribution of proteins of fast axonal transport following administration of $\beta, \beta^{\prime}$-iminodipropionitrile: A quantitative autoradiographic study. J. Cell Biol. 95: 672-675.

Rambourg, A., and B. Droz (1980) Smooth endoplasmic reticulum and axonal transport. J. Neurochem. 35: 16-25.

Requena, J. (1983) Calcium transport and regulation in nerve fibers. Annu. Rev. Biophys. Bioeng. 12: 237-257.

Rieder, C. L. (1981) Thick and thin serial sectioning for the three-dimensional reconstruction of biological ultrastructure. Methods Cell Biol. 22: 215-249.

Schlaepfer, W. W. (1974) Calcium-induced degeneration of axoplasm in isolated segment of rat peripheral nerve. Brain Res. 69: 203-215.

Schlaepfer, W. W., and R. P. Bunge (1973) Effects of calcium ion concentra. tion on the degeneration of amputated axons in tissue culturc. J. Cell Biol. 59: $456-470$.

Schlaepfer, W. W., U. J. P. Zimmerman, and S. Micko (1981) Neurofilament proteolysis in rat peripheral nerve. Homologous with calciurr-aclivated proteolysis of other tissues. Cell Calcium 2: 235-250.

Schliwa, M. (1984) Mechanisms of intracellular organelle transport. In Cell and Muscle Motility, J. W. Shay, ed., Vol. 5, pp. 1-82, Plenum Press, New York.

Schnapp, B. J., R. D. Vale, M. P. Sheetz, and T. S. Reese (1985) Single microtubules from squid axoplasm support bidirectional movement of organelles. Cell 40: 455-462.

Schnepf, E., K. Hausmann, and W. Herth (1982) The osmium tetroxidepotassium ferrocyanide (OsFeCN) staining technique for electron microscopy: A critical evaluation using ciliates, algae, mosses, and higher plants. Histochemistry 76: $261: 271$.

Smith, D. E. (1973) The location of neurofilaments and microtubules during the postnatal development of Clarke's nucleus in the kitten. Brain Res. 55: 41-53.

Specht, S. C. (1977) Axonal transport in the optic system of neonatal and adult hamsters. Exp. Neurol. 56: 252-264.

Teichberg, S., and E. Holtzman (19/3) Axonal agranular reticulum and synaptic vesicles in cultured embryonic chick sympathetic neurons. J. Cell Biol. 57: 88-108.

Tsukita, S., and H. Ishikawa (1976) Three-dimensional distribution of smooth endoplasmic reticulum in myelinated axons. J. Electron Microsc. 25: 141149.

Tsukita, S., and H. Ishikawa (1979) Morphological evidence for the involvement of the smooth endoplasmic reticulum in axonal transport. Brain Res. 174: 315-318.

Tsukita, S., and H. Ishikawa (1980) The movement of membranous organelles in axons. Electron microscopic identification of anterogradely and retrogradely transported organelles. J. Cell Biol. 84: 513-530.

Ungar, F., I. Piscopo, J. Letizia, and E. Holtzman (1984) Uptake of calcium by the endoplasmic reticulum of the frog photoreceptor. J. Cell Biol. 98 . $1645-1655$.

Walz, B. (1979) Subcellular calcium localization and ATP-dependent $\mathrm{Ca}^{2+}$ uptake by smooth endoplasmic reticulum in an invertebrate photoreceptor cell. An ultrastructural, cytochemical and X-ray microanalytical study. Eur. 
J. Cell Biul. 20: 83-91.

Walz, B. (1982a) Calcium-sequestering smooth endoplasmic reticulum in retinula cells of the blowfly. J. Ultrastruct. Res. 81: 240-248.

Walz, B. (1982b) $\mathrm{Ca}^{2+}$-sequestering smooth endoplasmic reticulum in an invertebrate photoreceptor. I. Intracellular topography as revealed by OsFeCN staining and in situ Ca accumulation. J. Cell Biol. 93: 839-848.

Walz, B. (1982C) $\mathrm{Ca}^{2+}$-sequestering smooth endoplasmic reticulum in an invertebrate photoreceptor. II. Its properties as revealed by microphotometric measirements. If. Cell Binl 93: 849-859

Walz, B. (1983) Association between cytoskeletal microtubules and $\mathrm{Ca}^{2+}$ sequestering smooth ER in Semper cells of fly ommatidia. Eur. J. Cell Biol. 32: 9298.

Weiss, D. G., V. Krygier-Brevard, G. W. Gross, and G. W. Kreutzberg (1978) Rapid axoplasmic transport in the olfactory nerve of the pike: II. Analysis of transpor led proteins by SDS gel electrophoresis. Brain Res. 139: 77-87.
Weiss, D. G., and G. W. Gross (1982) The microslrean hypothesis of axoplasmic transport: Characteristics, predictions and compatibility with data. In Axoplasmic Transport, D. G. Weiss, ed., pp. 362-383, SpringerVerlag, Berlin.

Yamamoto, H., K. Fukunaga, E. Tanaka, and E. Miyamoto (1983) $\mathrm{Ca}^{2+}$ - and calmodulin-dependent phosphorylation of microtubule-associated protein 2 and tau factor, and inhibition of microtubule assembly. J. Neurochem. 41: 1119-1125.

Yamauchi, T., and H. Fujisawa (1982) Phosphorylation of microtubule-associated protein 2 by calmodulin-dependent protein kinase (kinase II) which occurs only in the brain tissues. Biochem. Biophys. Res. Commun. 109: 975-981.

Zucker, R. S., and N. Stockbridge (1983) Presynaptic calcium diffusion and the time courses of transmitter release and synaptic facilitation at the squid giant synapse. J. Neurosci. 3: 1263-1269. 